\title{
A novel AXIN2 germline variant associated with attenuated FAP without signs of oligondontia or ectodermal dysplasia
}

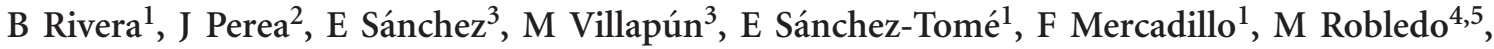 \\ J Benítez ${ }^{5,6}$ and M Urioste $e^{\star, 1,5}$
}

Truncating mutations in the AXIN2 gene, a key regulator of $\beta$-catenin degradation in the Wnt pathway, have been reported in three families with gastrointestinal adenomatous polyposis and features of ectodermal dysplasia. However, the role of AXIN2 in familial adenomatous polyposis (FAP) syndrome is not completely understood. We performed an in-depth study of $A P C$ and MUTYH, and ruled out their implication in 23 FAP families. We then investigated the role of other genes involved in the Wnt pathway, including AXIN2, and identified a novel missense variant in AXIN2 in one family with attenuated FAP. Carriers of the variant exhibited a variable number of polyps but none showed any sign of ectodermal dysplasia. We have demonstrated the pathogenicity of this novel variant by establishing its low frequency in controls as well as by LOH analysis, a segregation study, and immunofluorescent staining of AXIN2 and $\beta$-catenin proteins. This report expands the phenotype known to be related to AXIN2 alterations and raises the question of whether to screen AXIN2 in FAP cases negative for alterations in APC and MUTYH. European Journal of Human Genetics (2014) 22, 423-426; doi:10.1038/ejhg.2013.146; published online 10 July 2013

Keywords: familal adenomatous polyposis; AXIN2 mutation; oligodontia; ectodermal dysplasia; Wnt pathway

\section{INTRODUCTION}

Familial Adenomatous Polyposis (FAP) is a syndrome characterized by the development of hundreds of colorectal polyps in early adolescence; it predisposes sufferers to cancer and has dominant inheritance. Attenuated FAP (AFAP) is diagnosed when patients present with less (10-99) polyps, and tends to occur 15-20 years later than for classical FAP. Mutations in the APC gene are responsible for the majority of FAP cases, and also explain some AFAP cases. In 2002, the implication of MUTYH, a BER-pathway gene, was discovered in milder cases of polyposis. Nevertheless, a considerable proportion (20-50\%) of FAP and AFAP cases is not found to carry germline mutations in $A P C$ or $M U T Y H$, and their genetic etiology remains unclear.

As APC has a key role in maintaining the $\beta$-catenin turnover in the Wnt pathway, other Wnt genes involved in this regulation might be also implicated in the syndrome. It has been suggested that AXIN2, the scaffold protein of the $\beta$-catenin destruction complex, is the main negative regulator in the pathway. ${ }^{1}$ Little is known regarding the role of AXIN2 in CRC, but AXIN2 mutations have been reported in three families with gastrointestinal polyposis and ectodermal dysplasia;, ${ }^{2,3}$ the AXIN2 truncating mutations were located in a narrow region of the gene, and the phenotype associated with them, while highly heterogeneous, always included colonic polyposis and ectodermal dysplasia.

Here we describe a family with a novel missense variant in the AXIN2 gene, and thereby expand on the phenotype associated with alterations in this gene.

\section{METHODS}

FAP families were recruited as part of a project investigating the molecular basis of this syndrome. The project was approved by the ethics committee of the Instituto de Salud Carlos III and all patients provided informed consent. Twenty-three families were included in the present study because they tested negative for alterations in MUTYH and APC. The complete sequence of MUTYH was then studied by direct sequencing and MLPA. An extensive interrogation of $A P C$ involved direct sequencing of the gene, including the $1 \mathrm{~A}$ and $1 \mathrm{~B}$ promoters and the $3^{\prime} \mathrm{UTR}$ region, and assessing allelic imbalance. The Wnt genes AXIN2 and GSK3 $\beta$ were screened for mutations using dHPLC and direct sequencing.

In order to confirm the novel origin and deleterious nature of the variant, we consulted the Human Genetic Mutation Database (HGMD), Exome Variant Server, NHLBI GO Exome Sequencing Project (ESP), Seattle, WA (http://evs.gs.washington.edu/EVS/) [09, 2012] and 1000 Genomes databases. LOH was assessed by PCR amplifying and sequencing a 420-bp fragment surrounding the variant in tumoral versus germline DNA. Immunohistochemical (IHC) staining for AXIN2, APC and $\beta$-catenin was carried out using the ab32197 (Abcam, Cambridge, UK), sc896 (Santa Cruz, Heidelberg, Germany) and FLEX IR702 (Dako, Barcelona, Spain) antibodies, respectively. In order to evaluate the co-localization of AXIN2 and $\beta$-catenin, double staining of both proteins was performed by immunofluorescence (IF) and analyzed using a SP5-WLL confocal microscope (Leica, Barcelona, Spain).

Variant frequency was assessed by genotyping a control group of 800 individuals using the KASPar SNP genotyping system (KBioscience, Berlin, Germany). Allelic discrimination was carried out using the ABI PRISM 7900 Sequence Detection System (Applied Biosystems, Madrid, Spain). Evolutionary conservation of variants was evaluated as a probability using Phastcons (http://genome.cshlp.org/content/15/8/1034).

${ }^{1}$ Familial Cancer Clinical Unit, Spanish National Cancer Research Centre (CNIO), Madrid, Spain; ${ }^{2}$ General Surgery Service, Hospital 12 de Octubre, Madrid, Spain; ${ }^{3}$ Gastro-Endoscope Service, Clínica Ntra. Sra. Del Rosario, Madrid, Spain; ${ }^{4}$ Hereditary Endocrine Cancer Group, Spanish National Cancer Research Centre (CNIO), Madrid, Spain; ${ }^{5}$ Centro de Investigaciones Biomédicas en Red de Enfermedades Raras (CIBERER), Madrid, Spain; ${ }^{6}$ Human Genetics Group, Spanish National Cancer Research Centre (CNIO), Madrid, Spain

${ }^{*}$ Correspondence: Dr M Urioste, Familial Cancer Clinical Unit, Human Cancer Genetics Program, Spanish National Cancer Research Centre, Melchor Fernández Almagro, 3 , Madrid 28029, Spain. Tel: +34 917328000 (ext: 3315); Fax: + 3491224 6911; E-mail: murioste@cnio.es

Received 4 December 2012; revised 26 May 2013; accepted 6 June 2013; published online 10 July 2013 


\section{RESULTS}

A novel variant in AXIN2 was found in one family. The probandus was a male who developed 10 colorectal adenomas at age of 31 years. Eleven years later, he underwent a partial colectomy to treat CRC. Subsequent colonoscopies revealed 1-2 polyps every year. His sister was diagnosed with 30 polyps and CRC at age of 36 years. Their father died from CRC at the age of 51 years. None of these cases in the family had extracolonic manifestations associated with FAP. Since the family underwent ongoing surveillance by a clinical geneticist, an oncologist and a gastroenterologist, their clinic history was extensively reviewed, but no records of ectodermal dysplasia had been reported. A subsequent dermatological examination did not identify any dental abnormalities, hair scarceness, nail development problems or skin problems, and no features of oligodontia or ectodermal dysplasia were found in the probandus nor in any of his four sisters (Figure 1).

The novel variant c.1387C $>\mathrm{T}$; p.R463C was first found in the probandus. It is located in exon 5 , within the $\beta$-catenin binding domain, and it did not appear in the public databases (HGMD, NHLBI ESP and 1000 Genomes). The Condel predictor program (http://bg.upf.edu/condel/home) classified it as deleterious with a consensus score of 0.541 , although other predictor programs gave contradictory results. According to Phastcons, both the nucleotide and the domain were highly conserved through 46 vertebrates and 33 placental mammals, with a probability score of 1 . It was also found to be highly conserved across 10 species of primates, the only exception being Macaca mulata.

Two adenomas from the probandus and four polyps (two adenomas, one hyperplasic polyp and one mixed polyp with both adenoma and hyperplasic features) from one sister were available for further study of LOH, as well as IHC and IF staining. LOH of the mutated allele was identified in one polyp from the probandus'. Two polyps (one adenoma and the mixed polyp) from his sister (III-3, Figure 1) also presented $\mathrm{LOH}$, whereas other adenoma (with low-grade dysplasia) had reduced amplification of one allele, and therefore incipient LOH, but not sufficiently so to confirm LOH per se; her hyperplasic polyp showed no sign of $\mathrm{LOH}$.

Figure 2 shows APC, AXIN2 and $\beta$-catenin IHC expression in the adenoma from the probandus, and in polyps from an APC mutation carrier and a MUTYH mutation carrier. Double staining by using IF suggested that in comparison with normal crypts, the adenomatous gland showed higher levels of AXIN2 and $\beta$-catenin proteins (Figure 3). Moreover, AXIN2 was accumulated in the cytoplasm, whereas $\beta$-catenin was accumulated in the membrane and the nucleus, thus confirming the lack of co-localization of these two proteins. Finally, the c.1387C $>\mathrm{T}$ variant was not found in any of the 800 Spanish controls. Taken together, these results point to the possible pathogenicity of the variant; thus we performed a segregation study by testing for the variant in other family members. The affected sister also carried the variant. The mother of the probandus (aged 82 years) was found not to carry the variant, and therefore the father was considered an obligate carrier. Two apparently asymptomatic sisters (III-3 and 4, Figure 1), aged 51 and 46 years, were both noncarriers. Both underwent periodic surveillance by colonoscopy and no polyps were diagnosed. A fourth sister (III-5), aged 43 years, was found to be a carrier of the variant. She was provided with genetic counseling and underwent a colonoscopy that showed no evidence of polyps.

\section{DISCUSSION}

The c.1387C > T variant in AXIN2 may be related to the development of AFAP in this family. Studies carried out on tumoral tissue $\mathrm{LOH}$, IHC and co-localization assays) suggested a probable alteration of AXIN2 function. In addition, the variant appears to segregate with disease in the family, and it was not found in 800 Spanish controls nor reported in public databases, with information from over 13000 individuals.

Although knowledge about germline AXIN2 alterations and their associated phenotypes is limited, variants in the gene have been reported in cases with gastrointestinal neoplasia, with substantial

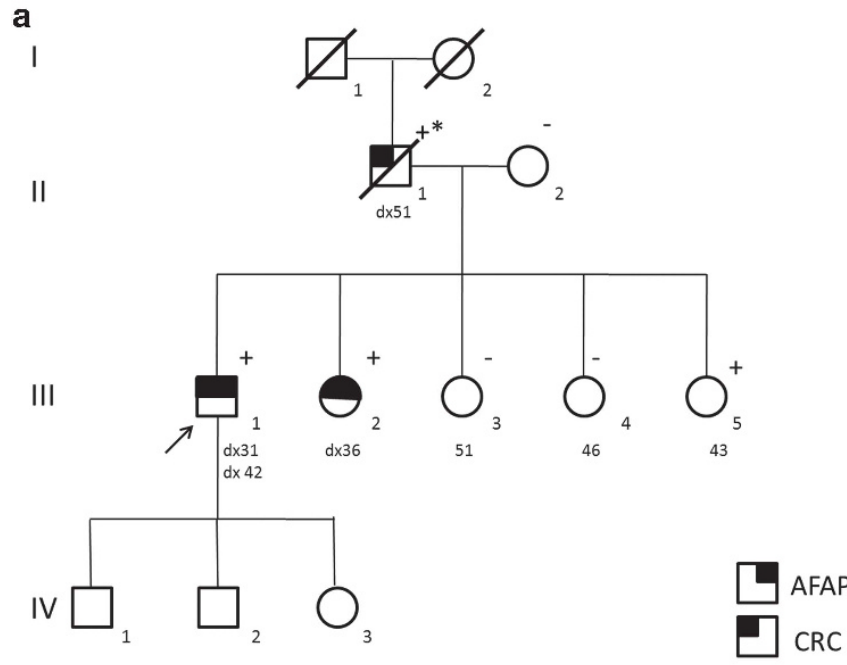

b

rs.1133683 $\downarrow \downarrow c .1378 C>T$

C TA T A G C C C T H G C T C C C G C T

rs.1133683 $\downarrow \downarrow$ c. $1378 C>T$ C TATA G C C CN TG C T C C G C T
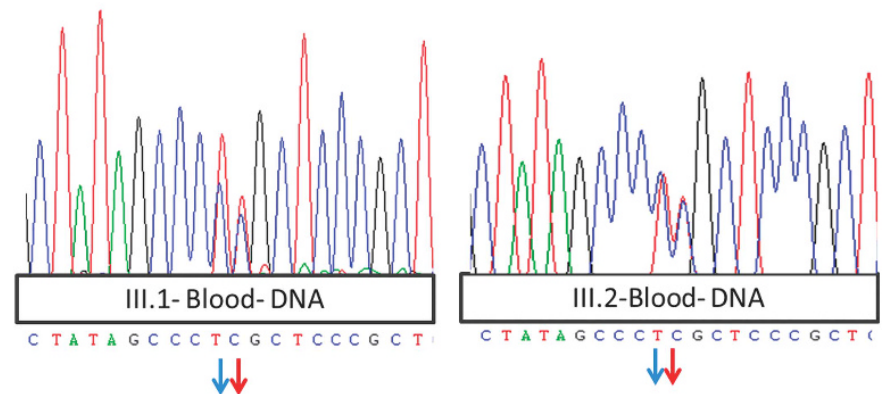

$\downarrow$
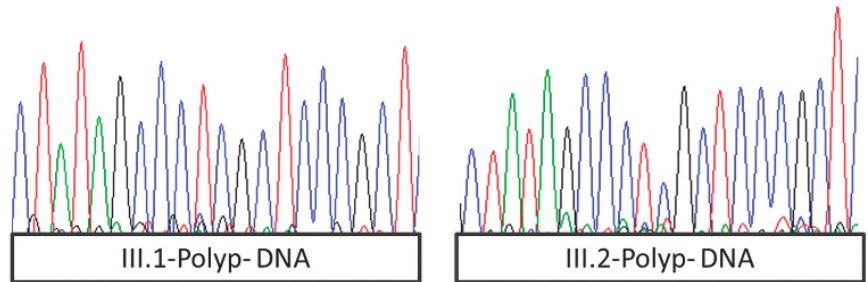

Figure 1 (a) Pedigree of the family. (b) LOH analysis in both carriers of the variant, individuals III.1 and III.2. Sequences of germline DNA from blood and polyp DNA from both affected individuals III.1 and III.2 are shown. Red arrows correspond to the variant c.1387C $>$ T; p.R463C found in both individuals; blue arrows mark rs1133683. 

NORMAL COLONIC MUCOSA

HE

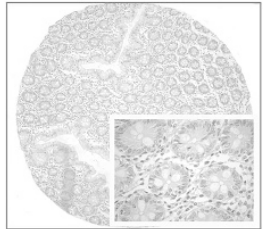

APC

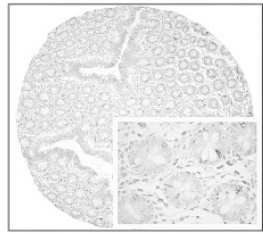

AXIN 2

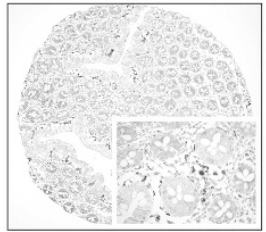

B CAT

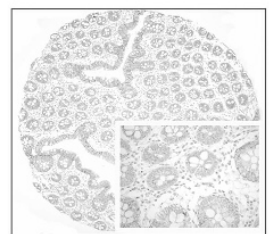

b PROBANDUS CASE: III.1
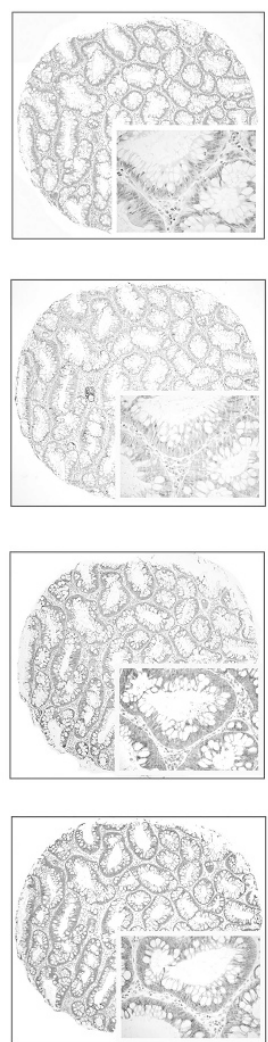

C CASE $A P C+$
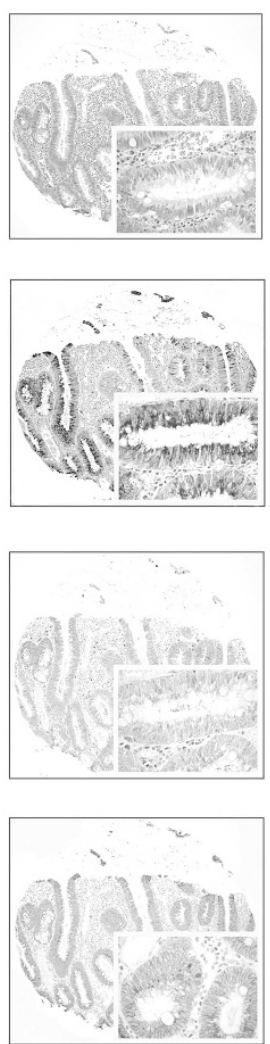

d
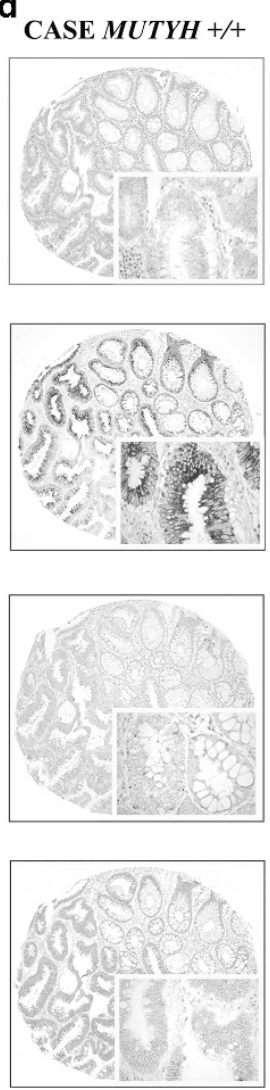

Figure 2 Immunohistochemical expression patterns of APC, $\beta$-catenin and AXIN2 proteins are shown in (a) normal colonic mucosa; (b) an adenomatous polyp of the probandus (III.1 Figure 1, carrier of the variant c.1387C >T; p.R463C); (c) an adenomatous polyp from an APC c.1959-2_1960dup;p.Ile 656AlafsX20 carrier and (d) an adenomatous polyp from a carrier of bialellic MUTYH mutations c.1187 G>A;p.Gly396Asp +c.1187G>A;p.Gly396Asp. AXIN2 overexpression was present in probandus compared with the APC and MUTYH mutation carriers. Moreover, APC overexpression was found in both APC and MUTYH mutation carriers but was absent in the probandus carrier of the c.1387C >T; p.R463C AXIN2 variant. HE: hematoxylin and eosin staining. Staining was performed using a Tissue Microarray; the inset corresponds to a $\times 600$ magnification. The full colour version of this figure is available at European Journal of Human Genetics online.

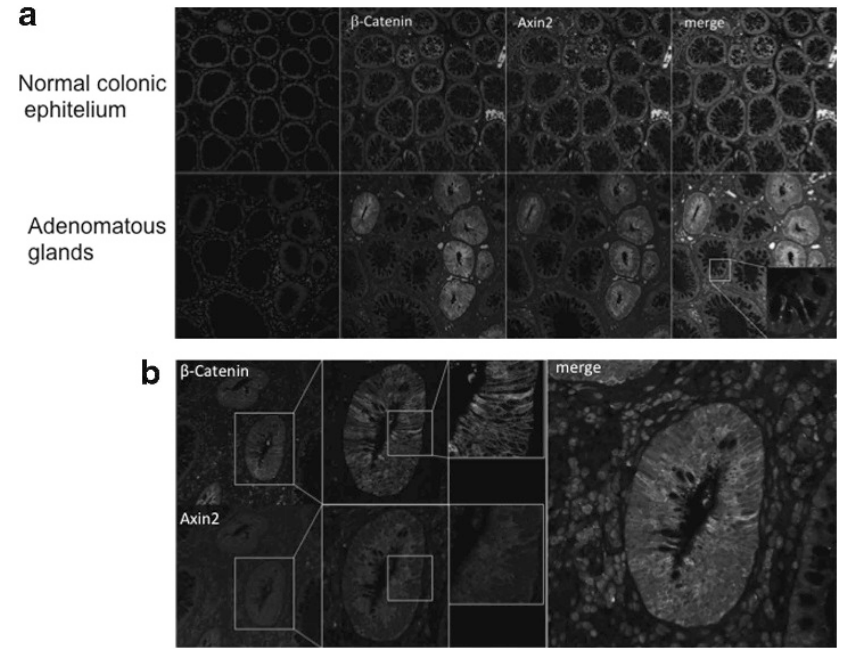

Figure 3 (a) AXIN2 and $\beta$-catenin overexpression in adenomatous polyp glands compared with paired normal colonic epithelium. The inset shows the results for a normal gland. (b) Lack of co-localization of AXIN2 and $\beta$-catenin proteins in an adenomatous polyp gland. The full colour version of this figure is available at European Journal of Human Genetics online. variability in the age of onset as well as in the number and type of polyps. For example, Lammi et $a .^{2}$ described a family with nine carriers of a nonsense mutation in AXIN2; the gastrointestinal phenotype in carriers varied from 68 adenomas in a 57-year-old carrier to no adenomas in a 26-year-old and one adenoma in a 58 -year-old. Even greater clinical variability was reported by Marvin et $a l,{ }^{3}$ in terms of the number (from none to more than 100), type (adenomatous or hyperplastic) and location (colonic or gastric) of polyps, and the presence of neoplasias. That no polyps were found in the 43-year-old variant-carrying sister of the probandus in the present study is consistent with this previously observed high clinical variability.

The lack in our variant-carrying family of signs of ectodermal dysplasia and of other extracolonic manifestations frequently found in FAP and AFAP families is also remarkable. The great majority of AXIN2 mutations have been identified in families selected because they had oligodontia, which likely introduced a selection bias into these studies. However, other conditions such as oral cleft and different types of cancer have been recently related to mutations in AXIN2., ${ }^{4,5}$ Although it is possible that AXIN2 has a role in colorectal tumor predisposition, few reports to date have tested the AXIN2 gene in CRC/poliposis patients. Leujene et al. ${ }^{6}$ studied 39 patients, one of 
whom had severe oligodontia, and only two variants in AXIN2 were found, both unlikely to be pathogenic. Peterlongo et al. ${ }^{7}$ reported several AXIN2 variants in a study of 82 patients with hereditary CRC, but none were considered pathogenic. In a study of cases negative for APC and MUTYH mutations, Renkonen et al., ${ }^{8}$ described an AXIN2 mutation in a family with previously reported oligodontia. ${ }^{2}$ Mongin et al. ${ }^{9}$ described 38 patients with adenomas or serrated polyps in which AXIN2 screening gave negative results. In light of these studies, it is likely that the number of polyposis patients who have undergone testing for AXIN2 alterations is still insufficient to define the role of the gene in familial polyposis.

With the limited number of AXIN2 mutations described to date, it is also difficult to establish a specific phenotype associated with a particular mutation. Nevertheless, all the mutations described in association with oligodontia are located in exon 7 of the gene, downstream of nucleotide 1900. The variant described here is located a substantial distance away, in exon 5. It has been described for other predisposition genes, such as $A P C$, that different phenotypes may present, depending on the protein domain affected by the mutation. The existence of second and third hits in an APC has been previously reported, and explained using the 'just right level of $\beta$-catenin' model. ${ }^{10}$ We have identified $\mathrm{LOH}$ of the mutated allele that would constitute a second hit event; taking into account the accumulation of AXIN2 protein in the cytoplasm, we speculate that a third hit event may occur in the wild-type allele, leading to a dominant-negative effect by the retained product. Therefore, further studies of larger patient series, as well as functional assays, are needed to clarify the role of AXIN2 in FAP.

In summary, we have identified a novel missense AXIN2 variant present in an AFAP family with no signs of oligodontia, ectodermal dysplasia or EM. This report potentially extends the phenotype caused by AXIN2 alterations and, though the associated phenotype is still not well understood, the testing of AXIN2 in FAP cases that are found to be negative for $A P C$ and $M U T Y H$ mutations may be an important clinical consideration.

\section{CONFLICT OF INTEREST}

The authors declare no conflict of interest.

\section{ACKNOWLEDGEMENTS}

This work has been partially supported by grant $n^{\circ} 10 / 00554$ from the Fondo de Investigaciones Sanitarias and the FPU Grant Program (Ministerio de Educación y Ciencia, Gobierno de España). We thank the Immunohistochemistry and Confocal Units, and the Tumour Bank at the CNIO, Dr Ivan Muñoz, Dra Soledad Alonso and Maika González-Neira.

1 Huang SM, Mishina YM, Liu S et al: Tankyrase inhibition stabilizes axin and antagonizes Wnt signalling. Nature 2009; 461: 614-620.

2 Lammi L, Arte S, Somer M et al: Mutations in AXIN2 cause familial tooth agenesis and predispose to colorectal cancer. Am J Hum Genet 2004; 74: 1043-1050.

3 Marvin ML, Mazzoni SM, Herron CM, Edwards S, Gruber SB, Petty EM: AXIN2 associated autosomal dominant ectodermal dysplasia and neoplastic syndrome. Am J Med Genet A 2011; 155A: 898-902.

4 Pedace L, Castiglia D, De Simone $\mathrm{P}$ et al: AXIN2 germline mutations are rare in familial melanoma. Genes Chromosomes Cancer 2011; 50: 370-373.

5 Menezes R, Marazita ML, Goldstein McHenry T et al: AXIS inhibition protein 2 , orofacial clefts and a family history of cancer. J Am Dent Assoc 2009; 140: 80-84.

6 Lejeune S, Guillemot F, Triboulet JP et al: Low frequency of AXIN2 mutations and high frequency of MUTYH mutations in patients with multiple polyposis. Hum Mutat 2006, 27: 1064 .

7 Peterlongo P, Howe LR, Radice $\mathrm{P}$ et al: Germline mutations of AXIN2 are not associated with nonsyndromic colorectal cancer. Hum Mutat 2005; 25: 498-500.

8 Renkonen ET, Nieminen P, Abdel-Rahman WM et al: Adenomatous polyposis families that screen APC mutation-negative by conventional methods are genetically heterogeneous. J Clin Oncol 2005; 23: 5651-5659.

9 Mongin C, Coulet F, Lefevre JH et al: Unexplained polyposis: a challenge for geneticists, pathologists and gastroenterologists. Clin Genet 2011; 81: 38-46.

10 Segditsas S, Rowan AJ, Howarth $\mathrm{K}$ et al: APC and the three-hit hypothesis. Oncogene 2009; 28: 146-155. 